\title{
In-plane tensile behavior of shape memory alloy honeycombs with positive and negative Poisson's ratio
}

\begin{abstract}
This work illustrates the manufacturing and tensile testing of a novel concept of honeycomb structures with hexagonal and auxetic (negative Poisson's ratio) topology, made of shape memory alloy core material. The honeycombs are manufactured using Nitinol ribbons having $6.40 \mathrm{~mm}$ of width and $0.2 \mathrm{~mm}$ of thickness. The ribbons were inserted in a special dye using cyanoacrilate to bond the longitudinal strips of the unit cells. Tensile tests at room temperature (full martensite) were performed on the honeycombs. Finite element models of the honeycomb assemblies under tensile loading have been developed using nonlinear beam elements from a commercial code. Good agreement is observed between numerical nonlinear simulations and the experimental results.
\end{abstract}

Keyword: Auxetic; Finite element; Honeycomb; SMA; Tensile loading 